\title{
Preliminary assessment of knee laxity after anterior cruciate ligament reconstruction using bone patellar tendon bone and hamstring graft
}

Chakra Raj Pandey, MD; Rajiv Baral, MS; Sunit Dahal, MS; Sushil Sharma,MS ;

Aayush Bajracharya, MS; Ram Thapa, MS

Department of Orthopedics, Grande International Hospital, Kathmandu, Nepal

Corresponding author:

Rajiv Baral, MS

Email: rajibaral66@gmail.com

Received 3 July 2019

Accepted 23 Sep 2019

\section{Introduction \\ Laxity after anterior cruciate ligament reconstruction is one of the troublesome problems lasting many years. Laxity can be tested by clinical and arthrometer preoperatively as well as postoperatively. The purpose of this study is to evaluate postoperative laxity in Nepalese population.}

\section{Materials \& methods}

This retrospective study was carried out in Grande International Hospital, Kathmandu. All cases which had completed six-months follow up and presented in outpatient department were included in the study. Laxity was tested by Karl Storz Arthrometer using anterior force in 20 degrees of knee flexion with maximum manual force by the same doctor. The statistical significance level was set at $\mathrm{p}=0.05$. Statistical analysis was done using SPSS 16.

\section{Results}

21 cases operated via arthroscopic method were eligible for the study. Mean age was $28.3 \pm 10.44$. There were $14(66.7 \%)$ male $7(33.3 \%)$ female. A total of eight cases of laxity (36.36\%) were identified - six males (75\%) and two females (25\%). Hamstring grafts accounted for three cases of laxity (37.5\%) while Bone Patellar Tendon Bone Graft (BPTB) accounted for five cases (62.5\%). The mean follow-up was one and half years. Hamstring and bone patellar tendon bone graft were used in $6(28.6 \%)$ and $15(71.4 \%)$ cases respectively.

\section{Conclusion}

Previous studies have concluded that $>3 \mathrm{~mm}$ of laxity difference is associated with difficulties in vigorous activities. However, our patients were comfortable in their daily activities without significant clinical outcome.

Key words: Knee laxity, Arthrometer

\section{Introduction}

Laxity after anterior cruciate ligament reconstruction (ACLR) is one of the troublesome problems lasting many years. Laxity can be tested by clinical examination (Lachman, Anterior Drawer Test and Pivot shift) and arthrometer preoperatively as well as postoperatively. KT 1000 arthrometer is considered a more precise tool for evaluating laxity. However, there remains significant inter-observer variability ${ }^{1}$. More than $5 \mathrm{~mm}$ of postoperative graft laxity constitutes a procedural failure ${ }^{2}$. However, controversies regarding previous statement are aplenty, and lack sound scientific fact. 
The influence of residual postoperative knee laxity on long-term patient outcome has not been thoroughly investigated ${ }^{3}$. Excellent functional knee score is the norm at all stages of follow-up despite a wide range of arthrometric laxity changes ${ }^{4}$. Meniscus injury may predispose to the occurrence of graft laxity and subsequent development of graft failure and osteoarthritic change. This may influence the Lysholm score ${ }^{5}$. The purpose of this study is to evaluate outcome associated with postoperative laxity as measured by Karl Storz Arthrometer

\section{Materials and method}

This retrospective study was carried out in Grande International Hospital. All cases which had completed six months after the surgery and presented in outpatient department between July 2018 to March 2019 were included in the study. Isolated $A C L, A C L$ associated with meniscus repair/partial or total meniscectomy were included in the study. Multiligamentous, isolated posterior cruciate ligament reconstruction cases were excluded from the study.

ACL reconstruction was performed by arthroscopic technique with BPTB (bone patellar tendon bone graft) and hamstring graft by two-incision technique. Twenty one cases were included in this study. The outcome was assessed by the Lysholm score. Laxity was tested by Karl Storz Arthrometer using anterior force in 20 degrees of knee flexion with maximum manual force by the same doctor. Over $3 \mathrm{~mm}$ in anterior translation when comparing both knees was considered as increased laxity ${ }^{6}$. Cases were subjected to a standardized questionnaire translated into Nepali (closed ended format type) in outpatient department or via phone to assess the Lysholm score. Mean and the standard deviation were used for descriptive data. Independent sample t test was used to compare means. The statistical significance level was set at $p=0.05$. Statistical analysis was done using SPSS 16.

\section{Result}

There were 21 cases (males 14, females 7) operated via arthroscopic method. Mean age was 28.3 \pm 10.44 . Follow-up ranged from 6 months to three years. There were a total of eight cases of laxity (36.36\%) - six males (75\%) and two females (25\%). Hamstring grafts accounted for three cases of laxity (37.5\%) while BPTB accounted for five cases (62.5\%). Hamstring and bone patella tendon bone graft were used in 6 (28.6\%) and 15 (71.4\%) cases respectively (table 1 ).

Table 1: Patient demographics

\begin{tabular}{|l|c|}
\hline \multicolumn{1}{|c|}{ Characteristics } & n (\%) \\
\hline Sex & $14(66.7)$ \\
\hline Male & $7(33.3)$ \\
\hline Female & $28.23 \pm 10.44$ \\
\hline Mean Age \pm SD & \\
\hline Surgery & $15(71.4)$ \\
\hline ACL repair BTB Graft & $6(28.6)$ \\
\hline ACL repair Hamstring Graft & \\
\hline
\end{tabular}

Our study showed patient with more than $3 \mathrm{~mm}$ laxity difference between two knees is not significantly associated with decrease in Lysholm scoring $(p=0.199)$. One case required revision surgery. Although Lysholm score is better with laxity less than 3 in both group, it is not statistically significant (Table 2,3).

Table 2: Lysholm score vs laxity

\begin{tabular}{|c|c|c|}
\hline Lysholm Score & Laxity & $\boldsymbol{p}$-value \\
\hline$\leq 3$ & $>3$ & \\
\hline $85 \pm 16.20$ & $70 \pm 25$ & 0.199 \\
\hline
\end{tabular}

\section{Discussion}

Arthrometer is an important tool to diagnose ligament injury and to assess the objective measurement of laxity after ACLR. Many researchers use KT1000 arthrometer to evaluate laxity ${ }^{5}$. This study aims to identify the consequences of laxity tested by arthrometer on clinical outcome after ACLR. Our study shows laxity difference less than three has excellent Lysholm score and does not change with time. These patients do not require additional surgery. They also have few complaints like incision site pain, catching sensation and mild pain during walking.

Table 3: Bone Patellar Tendon Bone graft vs Hamstring graft

\begin{tabular}{|c|c|c|c|c|c|c|}
\hline \multirow{3}{*}{ Characteristics } & \multirow{2}{*}{\multicolumn{2}{|c|}{$\begin{array}{l}\text { ACL reconstruction BPTB graft } \\
(n=12) \\
\text { Laxity }\end{array}$}} & \multirow{3}{*}{$p$-value } & \multirow{2}{*}{\multicolumn{2}{|c|}{$\begin{array}{c}\text { ACL reconstruction Hamstring } \\
\text { Graft }(n=4) \\
\text { Laxity }\end{array}$}} & \multirow{3}{*}{$p$-value } \\
\hline & & & & & & \\
\hline & $\leq 3$ & $>3$ & & $\leq 3$ & $>3$ & \\
\hline Lysholm Score & $83.75 \pm 16.85$ & $72.50 \pm 22.17$ & 0.347 & $95 \pm 0(n=1)$ & $66.67 \pm 33.29$ & 0.538 \\
\hline
\end{tabular}


We observed that a case of laxity difference of 3 $\mathrm{mm}$ required revision surgery after 3 months. This patient complained of pain on extension which was later diagnosed as medial synovial plica with nodule in right ACL. He also complained of pain during vigorous activity and climbing stairs. Plica release and trimming of the nodule was done arthroscopically. Bach et al. found $92 \%$ of the patients had a maximum manual difference $\leqslant 3$ $\mathrm{mm}$. Ninety-two percent had a negative pivot shift at follow-up and Lysholm score of 88 . The reoperation rate was $10 \%$ with a mild flexion contracture as the most common reason ${ }^{7}$. Harter demonstrated that the substitutes did not elongate significantly during the period of the study ${ }^{8}$. In our study, one patient in a regular visit asked for $x$-ray. Though $x$-ray shows tunnel widening, graft was anteriorly placed, his laxity difference was less than 3 and had excellent Lysholm score.

Ricardo et al. found no correlation between postoperative knee laxity and Lysholm score ${ }^{9}$. Our study also shows that patients with greater postoperative knee laxity are associated with poorer subjective knee outcome but it is not statistically significant ( $p=0.199)$. In a retrospective study by Kim et al. in thirty-one patients with generalized laxity and fifty two patients with normal laxity had a follow up 24 months found that side to side difference in anterior translation had an inverse correlation with Lysholm score in both groups ${ }^{10}$. The result of ACLR with a BPTB graft is superior to those associated with the use of a four bundle hamstring graft in patients with generalized laxity. Our study in the normal population indicates side to side differences had an inverse correlation with Lysholm score but it is not statistically significant. Sundemo et al. studied 193 patients and followed up 2-16yrs found long-term inferior subjective outcome associated with laxity ${ }^{3}$. Knee laxity is tested by Lachman, Anterior drawer, pivot shift and KT1000 arthrometer. However,the KT-1000 arthrometer result was not correlated with any outcome variables. Goodwillie et.al evaluated in 87 patients with 66 tight graft ( $<3 \mathrm{~mm}$ laxity) and 21 loose graft ( $>5 \mathrm{~mm}$ laxity). The procedure was performed via transtibial BPTB ACLR between 1992 to 1998 and followed up sixteen years. They suggested that $>5 \mathrm{~mm}$ laxity does not appear to place patients at a worse clinical outcome at longterm follow-up, nor does it lead to significantly more subsequent procedures ${ }^{2}$ which is similar to the study conducted by us. Goodwillie et al. also found a trend of increase additional procedure in tight grafts and one of the complications of lax graft is osteoarthritis changes after ACLR ${ }^{2}$. However we could not find increase rate of additional procedure in the tight graft. Moreover, we need long-term follow-up with serial radiograph to identify the occurrence of an osteoarthritic change. Our study showed that that ACL tear is associated with meniscus injury results in more laxity and inferior Lysholm score. Deleda et al. also concluded that instability and future progression to osteoarthritis is inevitable with $A C L$ reconstruction without meniscus repair ${ }^{11}$.

Alford et al. performed arthrometric analysis before and after surgery in patellar tendon group whereas we performed in both hamstring and BPTBgraft ${ }^{6}$. Bach etal. in their study in BPTB graft group with minimum follow-up of 2 years found similar results to this study ${ }^{7}$. Jia et al. could not find laxity more than $3 \mathrm{~mm}$ in their study using artificial ligament in the reconstruction of $\mathrm{ACL}^{5}$. Sundemo et al. in their study by using manual stress test without KT 1000 arthrometre found inferior long term outcome associated with increased laxity ${ }^{3}$. In contrast to the results of the study conducted by Harter et al. we used maximum manual force rather than specific value. In addition to that we could not find any case of extension lag in our study ${ }^{8}$. Deledda et al. in their study by using KT2000 arthrometer found not more than $2 \mathrm{~mm}$ difference in anterior laxity between normal right and left knee in 92 percent of cases ${ }^{10}$. Giannotti et al. conducted a study using KT1000 arthrometer and found excellent outcome despite having wide range of arthrometric laxity which is in contrast to our study 4 .

Limitations of our study are lack of radiographic data after ACLR, small sample size and short follow-up period. The measurement is taken at 6 month period may not correlate with the long term follow up data.

\section{Conclusions}

Laxity can be bothersome for patient and treating doctor. Previous studies have concluded that $>3 \mathrm{~mm}$ of laxity difference is associated with difficulties in vigorous activities. However, our patients were comfortable in their daily activities without significant clinical outcome. Laxity was independent of choice of graft - bone patellar tendon bone or hamstring. 


\section{References}

1. Daniel D, Malcom L, Losse G, Stone M, Sachs $R$, Burks R. Instrumented measurement of anterior laxity of the knee. J Bone Joint Surg. 1985;67(5):720-6.

2. Goodwillie AD, Shah SS, McHugh MP, Nicholas SJ. The effect of postoperative KT-1000 Arthrometer score on longterm outcome after anterior cruciate ligament reconstruction. Am J Sports Med. 2017;45(7):1522-8.

3. Sundemo D, Sernert N, Kartus J, et al. Increased postoperative manual knee laxity at 2 years results in inferior long-term subjective outcome after anterior cruciate ligament reconstruction. Am J Sports Med. 2018;46(11):2632-45.

4. G iannotti BF, Fanelli GC, Barrett TA, Edson C. The predictive value of intraoperative KT-1000 arthrometer measurements in single incision anterior cruciate ligament reconstruction. Arthroscopy. 1996;12(6):660-6.

5. Jia Z, Xue C, Wang W, Liu T, Huang X, Xu W. Clinical outcomes of anterior cruciate ligament reconstruction using LARS artificial graft with an at least 7-year follow-up. Medicine. 2017;96(14):e6568.

6. Alford JW, Bach BR. Arthrometric aspects of anterior cruciate ligament surgery before and after reconstruction with patellar tendon grafts. Techniques in Orthopaedics. 2005;20(4):421-38.

7. Bach BR, Jones GT, Sweet FA, Hager CA. Arthroscopy-assisted anterior cruciate ligament reconstruction using patellar tendon substitution. Two- to four-year followup results. Am J Sports Med. 1994;22(6):75867.

8. Harter RA, Osternig LR, Singer KM. Instrumented Lachmantestsfortheevaluation of anterior laxity after reconstruction of the anterior cruciate ligament. J Bone Joint Surg Am. 1989;71(7):975-83.

9. Cristiani R, Engström B, Edman G, Forssblad $M$, Stålman $A$. Revision anterior cruciate ligament reconstruction restores knee laxity but shows inferior functional knee outcome compared with primary reconstruction. Knee Surg Sports Traumatol Arthrosc. 2019;27(1):137-45.

10. Kim SJ, Kim TE, Lee DH, Oh KS. Anterior cruciate ligament reconstruction in patients who have excessive joint laxity. J Bone Joint Surg Am. 2008;90(4):735-41.

11. Deledda D, Rosso F, Cottino U, Bonasia $D E$, Rossi R. Results of meniscectomy and meniscal repair in anterior cruciate ligament reconstruction. Joints. 2016;3(3):151-57. 